\title{
Live Births after Fertility-saving Surgery in Ovarian Borderline Tumor and Oocyte Donation: Case Report
}

\author{
Maria do Carmo Borges de Souza ${ }^{1}$, Roberto de Azevedo Antunes ${ }^{1}$, Marcelo Marinho de Souza ${ }^{1}$, Ana Cristina \\ Allemand Mancebo ${ }^{1}$, Patricia Cristina Fernandes Areas ${ }^{1}$, Euridice Maria de Almeida Figueiredo²
}

\author{
${ }^{1}$ FERTIPRAXIS - Reprodução Humana, Rio de Janeiro, RJ, Brazil \\ ${ }^{2}$ Instituto Nacional de Cancer (INCA), RJ, Brazil
}

\begin{abstract}
A left borderline serous ovarian tumor stage I (FIGO) was discovered and treated in a 26-year-old black nulliparous woman, by conservative approach (laparotomy, salpingo-oophorectomy). In a six months interval she had a 7.5 $\times 7.1 \times 5.7 \mathrm{~cm}$ multilocular contralateral tumor with septa and vegetative areas and in a year interval a CT showed a $8.4 \times 7.4 \times 7.0$ lesion that precluded a follicular aspiration. The authors discuss the multidisciplinary strategy and the approach with the couple: the best option considered would be the resection of the tumor remaining attached to a new study pelvic. If possible uterine conservation should be held for further procedure of oocyte donation. Two years from the first surgery she had the second laparotomy and six months later she had an ICSI with oocyte donation. She became pregnant and delivered two 34 week-pregnancy boys through $\mathrm{C}$-section due to hypertension plus preeclampsia. There happened a post- operative intestinal obstruction that required a new surgical approach to adhesions lysis. There was no report of tumor lesions then. Seven months later, mother and children are doing well. Comments are made about borderline ovarian tumors and fertility-sparing approaches.
\end{abstract}

Keywords: Ovarian cancer, Conservative surgery, ART, Oocyte donation.

\section{INTRODUCTION}

Cancer survivors are increasing and assisted reproductive technologies (ART) developments are also more common. So, fertility preservation of the reproductive-age women with cancer is emerging as a challenging but rewarding application of ART. Ovarian tumors diagnosed in young ages tend to be low-stage low-grade malignancies (Kleine, 1996). Although fertility saving surgery is more often confined to early-stage and low-grade disease, it also has been reported in advanced stages (up to Stage IIIc). Management of borderline ovarian tumors has evolved significantly in the last few decades and in contrast to invasive ovarian cancer, they can be operated on conservatively at all stages (Ayhan et al., 2003).

\section{Case description}

TPMS, 26-year-old nulliparous black woman and her husband were referred by the oncologic surgeon to the Fertility Clinic with the request to be assessed in relation to pregnancy. Husband 28 year-old, healthy man. Both nurses with university degree.

First consultation on 5 April 2012.

Reports irregular cycles and oncologic surgery in June 7, 2011. No contraception method at least 2 years.

Good general condition, $1.64 \mathrm{~m}$, weight $64 \mathrm{~kg}$, BMI 23.8. Normal blood pressure. Do not smoke, do not use medications. Family history of hypertension and myocardial infarction.
Operated at INCA (Brazilian National Cancer Institute) in June 7,2011 , diagnosis of borderline left ovarian tumor, held unilateral adnexectomy: $8.0 \times 7.0 \times 4.0 \mathrm{~cm}$ mass, $220 \mathrm{~g}$, with microinvasive foci. The greater omentum had one non-invasive implant, free diaphragmatic cupula and peritoneum. It was a Stage I BOT (FIGO, 2012) and she was advised to a careful follow-up.

On December 6, 2011 a transvaginal US control showed a right ovary cyst of $8.2 \times 5.4 \mathrm{~cm}$, with septa and mixed content and on March 20, 2012 this image was described as $7.5 \times 7.1 \times 5.0 \mathrm{~cm}$ and blood CA 125 test was $48.6 \mathrm{UmL}$. In a joint discussion with her oncologic surgeon it was established the technical difficulty of oocyte retrieval with this mass, after a new sonography evaluation performed at the clinic on this day consultation. The best option fertility-saving procedure considered would be the resection of the tumor remaining, attached to a new study pelvic. If possible, uterine conservation should be held for further procedure of oocyte donation.

A CT scan of the abdomen and pelvis was performed on May 16,2012 , which showed a right complex 8.4 x 7.0 $x 7.2 \mathrm{~cm}$ multilocular formation, with preserved uterus. By own difficulties of the couple and/or public hospital schedule a new CT scan was performed at INCA on December 11,2012 , a year and a half after the first operation, showing a progressive increase in tumor mass, now $12.0 \times 10,5$ $x 9.0 \mathrm{~cm}$.

On February 6, 2013 the couple returned to the clinic and it was agreed that we would begin the search process for a possible donor, according to the conditions prevailing in the country (CFM resolution, 2010). Solved the operation, with the approval of the Oncologic team, we would proceed to the treatment of assisted reproduction. At this time she was beginning antihypertensive drugs due to changes in blood pressure.

The second operation was held on July 9, 2013: Right adnexectomy, a tumor of $380 \mathrm{~g}, 14.0 \times 11 \times 8.5 \mathrm{~cm}$, reddish-brown outer surface, with an area of rupture exposing papillary lesion. Open, multilocular cystic cavity with papillary appearance in $50 \%$ of the area. Tube attached, congested and distended. Adhesions undone in the intestinal wall. Right parieto-colic gutter with non-invasive implant. Conclusion: serous borderline tumor with micro invasive foci, non-committed capsule, no special tube remarks. On December 16, 2013, two and a half years after the first surgery and 6 months after the second, we proceeded to ICSI cycle with donor oocytes. All couple exams were adequate, according to the country standards (CFM Resolution, 2010). No male factor.

The protocol for hormonal treatment for the recipient (the Case) was kept as usual. She had been previously taking Cicloprimogyna ${ }^{\circledR}$ (Schering, estradiol valerate $(E V)+$ levonorgestrel) and then was synchronized with the donator stimulating cycle. The starting standard dose of $4 \mathrm{mg}$ daily was increased to $6 \mathrm{mg}$ on the donor' s HCG day. Serial vaginal scans were performed during the treatment cycle. 
On the day of recovery of the donated oocytes, recipient began intravaginal Progesterone (Utrogestan $\AA$ Besins), 200 mg vaginal each 8 hours plus AAS infantil ${ }^{\circledR}$ (salicylic acid $100 \mathrm{mg}$ per day).

Seven metaphase II oocytes were injected with the Case's husband spermatozoa, with normal fertilization of 4 . On day 3 two embryos were transferred ultrasound guided (8CG1 and 9CG1), and one was vitrified, 13CG1. From this day on she increased EV dosis to $8 \mathrm{mg} /$ day, and the medications were kept until 10 weeks. From this point on she decreased EV to $6 \mathrm{mg}$ for 2 weeks, then to $4 \mathrm{mg}$ and stopped in another two weeks.

Resulted a twin dichorionic / diamniotic pregnancy, which evolved until the 34th week, when it was interrupted by raked hypertension and preeclampsia. A C-section was held. The 2 boys received an Apgar score of 8 and 9, with $1990 \mathrm{~g} / 42 \mathrm{~cm}$ and $2195 \mathrm{~g} / 45 \mathrm{~cm}$.

The mother presented a post- operative intestinal obstruction that required a new surgical approach to adhesions lysis. There was no report of tumor lesions then. Seven months later, mother and children are doing well and the couple declares they are happy and pleased to have been met.

\section{DISCUSSION}

The possibilities offered by in vitro-fertilization have enlarged the spectrum of fertility preserving surgery. So, conservative surgeries, now referred as fertility saving surgeries can be performed safely in germ cell, borderline and early stage epithelial ovarian tumors in selected cases as pointed to many authors (Chhabra \& Kutchi, 2013).

Borderline ovarian tumors (BOT) are neoplasms of controversial biologic potential and clinical significance, and appear to share a risk profile similar to that of malignant ovarian tumors. They tend to occur at younger ages (as our patient), and are associated with a much better prognosis. They account for ten to 20 percent of all epitelial ovarian carcinomas. Ozalp et al. (2014) related 28 patients younger than 40 years with BOT that underwent fertility sparing surgery (median follow up time was $42 \pm 28.1$ months). During the follow up period, two patients $(7.1 \%)$ developed recurrence at 35 and 36 months, respectively. Five $(17.9 \%)$ out of 28 patients became pregnant during the follow up period.

Park et al. (2009) compared BOT groups that underwent radical or fertility-sparing surgery, defined as the preservation of the uterus and ovarian tissue in one or both adnexa (from 360 patients with BOT , 344 had stage I, one had stage II, and 15 had stage III disease). A total of 184 underwent fertility-sparing surgery (48 laparoscopically and 136 laparotomically). After surgery, 45 patients received adjuvant chemotherapy. The recurrence rate was similar in the radical and fertility-sparing surgery groups ( $4.9 \%$ vs. $5.1 \%, P=0.923)$. In the fertility-sparing surgery group, however, the most common site of recurrence was the remaining ovarian tissue which was successfully salvaged with a second round of fertility-sparing surgery. They reported 34 full-term deliveries by women in their fertility-sparing surgery group.

Chen et al. (2014) in a retrospective analysis of the clinical outcomes in 12 recurrent BOT patients with second fertility-sparing surgery had the average onset age of 26 years, and none had procreation before the second operation. The pathological diagnoses were still BOT and the recurrent sites of 10 patients were still restricted to the ovaries, like our patient. Among 6 cases that desired for pregnancy after the second operation, 4 were successful. The spontaneous pregnancy rate was $4 / 6$.

Our patient presented a very early recurrence (6 months) and when she first came to the clinic it was not possible to consider a technical pick-up. It also too long to get to the second operation ( 2 years) and even though the keeping of the uterus was considered after thoroughly discussed with the oncologist. Although serous microscopic implants (microinvasion) were detected in the first surgery on compromised ovary, the uterine peritoneum is a small part of all pelvic peritoneum that was free. Also, in literature we could find individual authors proposing the conservation of the uterus after bilateral oophorectomy, keeping the option of a pregnancy by oocyte donation an option for the patient (Navot et al., 1991; Kleine,1996; Poully \& Alliassa, 1996). We could find no published report of live births in this situation as described in this report of ours.

Chen et al. (2014) had the mean duration of follow-up of 53 months (range: $23-97$ months) and there happened three new recurrences $(25 \%)$. Although no disease related deaths occurred a careful follow-up was indicated (the Case is now with a follow-up of 44 months). This is a point our patient will have to observe, including a potential hysterectomy, although again controversial (Patrono et al., 2013).

Our report serves to confirm that fertility-sparing approaches are well tolerated not only in patients with early-stage BOTs (like this Case) but also in patients with advanced-stage BOTs with noninvasive extraovarian implants, if these implants can be resected completely, as stated by Nam (2010).

There remain challenging issues about improving attention to long-term health and quality of life for patients facing cancer survival therapy during their reproductive years that should be incorporated in health care. A broad focus on these multidisciplinary approaches in this case report resulted in definitely a "fertile" association.

\section{CONFLICT OF INTERESTS}

No conflict of interest have been declared.

\section{Corresponding author:}

Maria do Carmo Borges de Souza

FERTIPRAXIS - Reprodução Humana

Rio de Janeiro, RJ, Brazil

E-mail: mariadocarmo@fertipraxis.com.br

\section{REFERENCES}

Ayhan A, Celik H, Taskiran C, Bozdag G, Aksu T. Oncologic and reproductive outcome after fertility-saving surgery in ovarian cancer. Eur J Gynaecol Oncol. 2003; 24:223-32.

CFM - Conselho Federal de Medicina. Resolução CFM 1957- 2010. D.O.U. 06 de janeiro de 2011, Seção I, p.79). http://www.portalmedico.org.br/resolucoes/ CFM/2010/1957_2010.htm. Access in Jan 29, 2014.

Chen R, Zhu T, Yu H, Zhu J, Lu X. [Retrospective analysis of the clinical outcomes in 12 recurrent borderline ovarian tumor patients with second fertility-sparing surgery]. Zhonghua Fu Chan Ke Za Zhi. 2014;49:254-9.

Chhabra S, Kutchi I. Fertility preservation in gynecological cancers. Clin Med Insights Reprod Health. 2013; 7:49-59.

FIGO Ovarian Cancer Staging. https://www.sgo.org/ wp-content/uploads/2012/09/FIGO-Ovarian-Cancer-Staging_1.10.14.pdf. Access on Jan 29, 2014.

Kleine W. [Results of fertility preserving operations in malignant ovarian tumors. Zentralbl Gynakol]. 1996; 118:317-21. 
Nam JH. Borderline ovarian tumors and fertility. Curr Opin Obstet Gynecol. 2010 22:227-34.

Navot D, Fox J, Williams M, Brodman M, Friedman F Jr, Cohen $\mathrm{CJ}$. The concept of uterine preservation with ovarian malignancies. Obstet. Gynecol. 1991; 78:566-8.

Park JY, Kim DY, Kim JH, Kim YM, Kim YT, Nam JH. Surgical management of borderline ovarian tumors: The role of fertility-sparing surgery. Gynecol Oncol. 2009; 113: 75-82.

Patrono MG, Minig L, Diaz-Padilla I, Romero N, Moreno JFR,
Garcia-Donas J. Borderline tumours of the ovary, current controversies regarding their diagnosis and treatment. Ecancermedicalscience. 2013;7:379.

Poully JL, Alliassa B. Cancer de I'ovaire et reproduction: grossesse après cancer, cancer et grossesse, traitement de la stérilité et risque de cancer. In Dauplat J, Guastella JP eds. Cancer de I'Ovaire. Paris: Blackwell Editions; 1996. p.623-45

Ozalp SS, Yalcin OT, Telli E, Oge T, Kabukcuoglu S. Borderline ovarian tumors: outcomes of fertility sparing surgery. Eur J Gynaecol Oncol. 2014; 35:154-6. 